\title{
Temperature issues with white laser diodes, calculation and approach for new packages
}

\author{
Roland Lachmayer*, Gerolf Kloppenburg, Serge Stephan \\ Leibniz Universität Hannover, Institute of Product Development, Welfengarten 1A, 30167 \\ Hannover, Germany
}

\begin{abstract}
Bright white light sources are of significant importance for automotive front lighting systems. Today's upper class systems mainly use HID or LED light sources. As a further step laser diode based systems offer a high luminance, efficiency and allow the realization of new dynamic and adaptive light functions and styling concepts.

The use of white laser diode systems in automotive applications is still limited to laboratories and prototypes even though announcements of laser based front lighting systems have been made. But the environment conditions for vehicles and other industry sectors differ from laboratory conditions. Therefor a model of the system's thermal behavior is set up.

The power loss of a laser diode is transported as thermal flux from the junction layer to the diode's case and on to the environment. Therefor its optical power is limited by the maximum junction temperature (for blue diodes typically $125-150{ }^{\circ} \mathrm{C}$ ), the environment temperature and the diode's packaging with its thermal resistances. In a car's headlamp the environment temperature can reach up to $80^{\circ} \mathrm{C}$. While the difference between allowed case temperature and environment temperature is getting small or negative the relevant heat flux also becomes small or negative. In early stages of LED development similar challenges had to be solved. Adapting LED packages to the conditions in a vehicle environment lead to today's efficient and bright headlights. In this paper the need to transfer these results to laser diodes is shown by calculating the diodes lifetimes based on the presented model.
\end{abstract}

Keywords: Laser, Automotive lighting, Laser activated remote phosphor, Thermal management of laser diodes, Lifetime modeling

\section{INTRODUCTION}

An increase of luminous efficiency of bright white LEDs over the past years has led to a rapidly growing number of LED systems being used for general lighting applications. Also in automotive front lighting, LEDs are replacing halogen lamps and Xenon discharge lamps. Typically, the white light of the LED is generated by covering a blue chip with a phosphor coating ${ }^{[1]}$. A part of the emitted blue light is then absorbed by the phosphor and converted to a spectrum band from green to red. The rest of the emitted light stays unconverted and is then mixed with the converted spectrum. The balance between converted and unconverted light as well as the chemical mixture of the phosphor allow the generation of a desired color temperature. Advances in LED systems' packaging have made very bright white light sources consisting of a single LED chip possible by optimizing the thermal coupling of the elements.

Laser diodes are technologically similar to an LED and the interest to develop high power laser diode systems is constantly rising. One of the reasons for this is the possibility to use a focused high-energy beam for optical setups and thus build smaller and lighter optics. It is also possible to build systems with a remote phosphor which allow an improved thermal management because there are two separated heat sources ${ }^{[2]}$. This kind of white light sources is already integrated in various models of multimedia projectors. There are three general approaches to generate white light from laser diodes. Two of them are based on the (remote) phosphor technology and one is to add beams of different wavelengths. The principles are shown in Figure 1.

*lachmayer@ipeg.uni-hannover.de; phone: +49 5117623471

Photonics, Devices, and Systems VI, edited by Pavel Tománek, Dagmar Senderáková, Petr Páta, Proc. of SPIE Vol. 9450, 94501P · (c) 2015 SPIE · CCC code: 0277-786X/15/\$18 · doi: 10.1117/12.2070411 


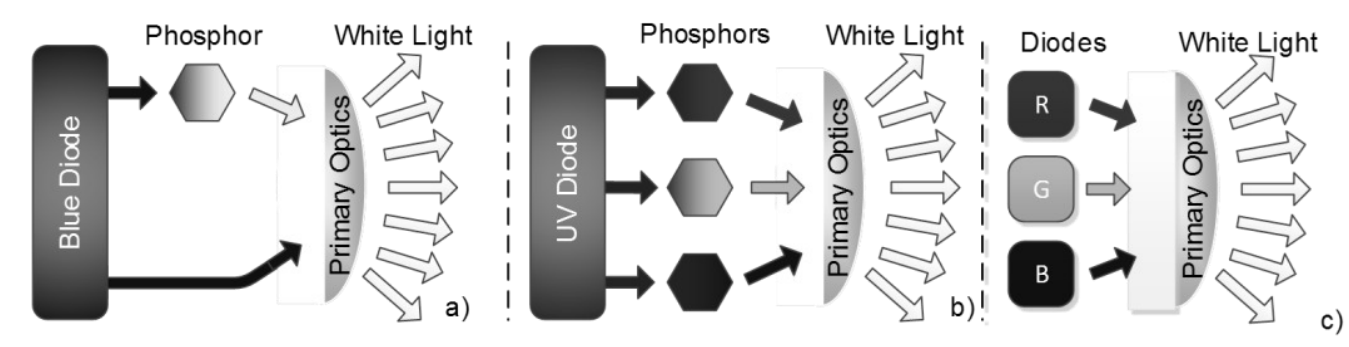

Figure 1. Scheme of laser based white light sources: a) mixing direct blue light and activated phosphors; b) converting total output of diode; c) mixing of different diodes (RGB-laser) according to ${ }^{[2]}$

When using LED or laser diodes for lighting applications usually multiple diodes have to be integrated into one system including electrical power supply and output control. Usually these systems consist of many parts that will cause the whole system to stop working when one element is damaged. Because of this, lifetime considerations for each component are a very important part in the development process of semiconductor systems.

\section{LIFETIME CALCULATIONS}

Lifetime calculations for laser diode systems can be done similar to LEDs. The method has been presented already in ${ }^{[3]}$. When using lifetime information about laser diodes from data sheets, they are usually referring to a case temperature of $25{ }^{\circ} \mathrm{C}$ at nominal current. The damage processes of LED and laser diode chips are similar and as a result the nominal lifetimes are comparable. Values given for laser diodes are around 30,000 hours. Increasing operating temperatures lead to a decrease in theoretical lifetime. To calculate the lifetime $L_{n}$ at a temperature level $T_{n}$, the following equation based on Arrhenius' description of the temperature dependence of reaction rates can be used ${ }^{[4]}$ :

$$
L_{n}=L_{r e f} \cdot e^{\left(\frac{E_{a}}{k_{B}}\left(\frac{1}{T_{n}}-\frac{1}{T_{r e f}}\right)\right)}
$$

With: $E_{a}$ : Activation Energy, $k_{B}$ : Boltzmann Constant, $T_{r e f}$ : Reference Temperature, $L_{r e f}$ : Measured Lifetime at Temperature $T_{r e f}$, the exponential term is called acceleration factor $b$.

$$
L_{n}=L_{r e f} b
$$

Operating temperatures may vary during the product usage period. This is caused by changing environmental conditions as well as different operating currents. For automotive applications the specified environment temperature range is from $-40{ }^{\circ} \mathrm{C}$ to $+80{ }^{\circ} \mathrm{C}$. The same light sources are often used for various lighting functions such as tail light and breaking light. In this case different operating conditions and thus thermal loads occur. These actual operating conditions have to be taken into account to approximate the diode's lifetime.

The total real lifetime of a laser diode can be split at into $\Delta \tau_{i}$ time intervals with constant temperatur loads $T_{i}$.

$$
L_{n}=\sum_{i=1} \Delta \tau_{i}
$$

The damage equivalent time for operation at $T_{r e f}$ in $\Delta \tau_{i}$ can be calculated by

$$
\Delta t_{i}=\Delta \tau_{i} b_{i}^{-1}
$$

If $\Delta t_{i}$ sum exceeds the reference lifetime, then the laser diode is permanently damaged. Equation (5) shows a context of this.

$$
L_{r e f} \geq \sum_{i=1} \Delta \tau_{i} b_{i}{ }^{-1}
$$

For infinitesimal small time elements $\Delta \tau_{i}$ the sum can be replaced by an integral. 


$$
L_{r e f} \geq \int_{0}^{t} e^{\left(-\frac{E_{a}}{k_{B}}\left(\frac{1}{T(\tau)}-\frac{1}{T_{r e f}}\right)\right)} d \tau
$$

Is the function of $T(\tau)$ thus the maximm new lifetime can bin predicted. Or is it possible to estimate lifetime at any time point to the lifetime end.

\section{SYSTEM EFFICIENCY AND LONG TERM BEHAVIOR}

The system set up in chapter 4.3 is used to determine the long term behavior of the white laser light source. Regarding an automotive application, the light source has to achieve a minimum lifetime of 1000 hours. For the use of the laser system as a low beam light source, a luminous flux of 1000 lumens has to be generated. The previously built light source has an optical output of $200 \mathrm{~lm}$ at $1200 \mathrm{~mA}$ input current and a case temperature of $25^{\circ} \mathrm{C}^{[4]}$. So theoretically an amount of 5 laser diodes is necessary to achieve the optical output specified. The thermal resistance between junction layer and case is a crucial value for the system's thermal behavior. For the laser diodes used it is $15 \mathrm{~K} / \mathrm{W}$. Due to the chip design and manufacturing processes of the diode, this property can only be optimized by the manufacturer. In comparison today's automotive LEDs achieve resistances of about $2 \mathrm{~K} / \mathrm{W}$ (see Table 1).

In Figure 2 a comparison of wall-plug efficiencies for selected LEDs and the white laser diode system is shown. To calculate the wall-plug efficiency, electrical input power $\mathrm{P}_{\mathrm{el}}$ of the system and the total luminous flux $\Phi$ in lumens is used. The datasheets of LEDs give information about the change of input voltage at different temperatures for a controlled input current as well as the corresponding change in the luminous flux. Values for the laser diode system are measured according to the description in section 4.3.

Table 1. Comparison of white diodes for automotive applications (Values from data sheets, except for luminous flux of No. 7) $[5][6][7][8],[9][10]$

\begin{tabular}{|c|l|c|c|l|}
\hline No. & \multicolumn{1}{|c|}{ Diode } & Max. $\mathbf{T}_{\mathbf{j}}\left({ }^{\circ} \mathbf{C}\right)$ & $\begin{array}{l}\text { Max Thermal } \\
\text { Resistance Rjc }\end{array}$ & \multicolumn{1}{c|}{ Luminous Flux } \\
\hline 1 & $\begin{array}{l}\text { OSLON Black Flat } \\
\text { KW H2L531.TE }\end{array}$ & 150 & $1.9 \mathrm{~K} / \mathrm{W}$ & $\begin{array}{l}500 \ldots 800 \mathrm{~lm} \\
@ 1000 \mathrm{~mA}\end{array}$ \\
\hline 2 & $\begin{array}{l}\text { OSLON Black Flat } \\
\text { LUW H9QP }\end{array}$ & 150 & $7.5 \mathrm{~K} / \mathrm{W}$ & $\begin{array}{l}180 \ldots 280 \mathrm{~lm} \\
@ 700 \mathrm{~mA}\end{array}$ \\
\hline 3 & $\begin{array}{l}\text { OSRAM OSTAR } \\
\text { LE UW U1A3 01 }\end{array}$ & 150 & $3.6 \mathrm{~K} / \mathrm{W}$ & $\begin{array}{l}630 \ldots 1120 \mathrm{~lm} \\
@ 1000 \mathrm{~mA}\end{array}$ \\
\hline 4 & $\begin{array}{l}\text { OSRAM OSTAR } \\
\text { LE UW U1A5 01 }\end{array}$ & 150 & $2.5 \mathrm{~K} / \mathrm{W}$ & $\begin{array}{l}1120 \ldots 1800 \mathrm{~lm} \\
@ 1000 \mathrm{~mA}\end{array}$ \\
\hline 5 & $\begin{array}{l}\text { PHILIPS LXMA- } \\
\text { PW01-0110 }\end{array}$ & 150 & $10 \mathrm{~K} / \mathrm{W}$ & $\begin{array}{l}199 \mathrm{~lm} \\
@ 700 \mathrm{~mA}\end{array}$ \\
\hline 6 & $\begin{array}{l}\text { PHILIPS LXMA- } \\
\text { PW01-0130 }\end{array}$ & 150 & $15 \mathrm{~K} / \mathrm{W}$ & $\begin{array}{l}233 \mathrm{~lm} \\
@ 700 \mathrm{~mA}\end{array}$ \\
\hline 7 & $\begin{array}{l}\text { OSRAM } \\
\text { PL TB450B } \\
(+ \text { phosphor added) }\end{array}$ & 150 & $\begin{array}{l}205 \mathrm{~lm} @ 1200 \mathrm{~mA}, \\
\mathrm{~T}_{\text {case }}=20^{\circ} \mathrm{C}\end{array}$ \\
\hline
\end{tabular}




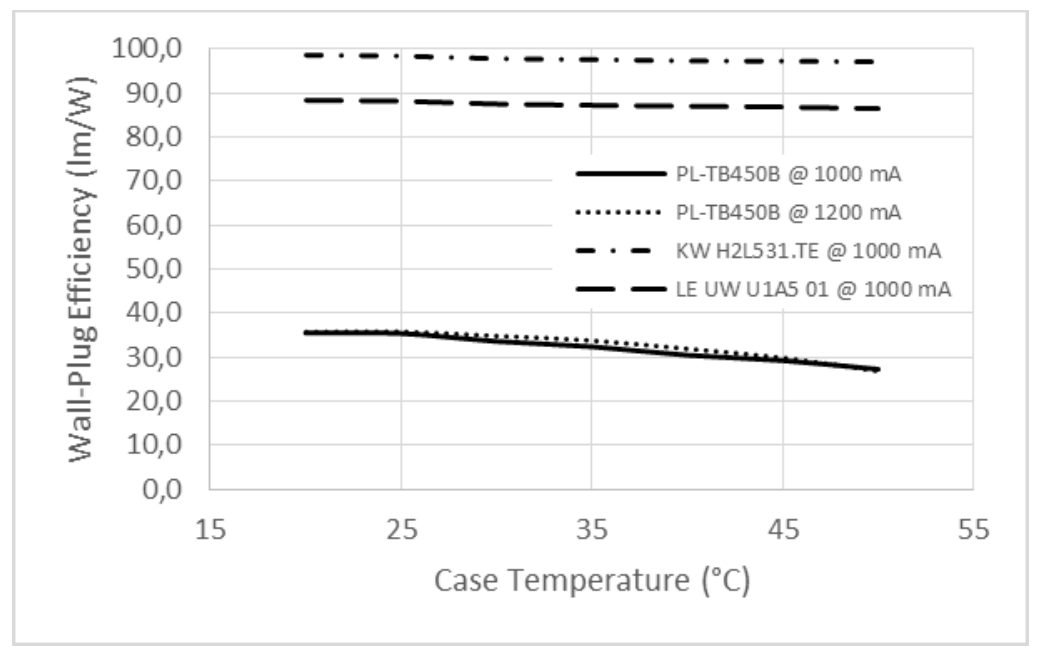

Figure 2. Wall-Plug Efficiency of a laser activated remote phosphor system at different case temperatures

Using two LED sources (No. 1 and 4) from Table 1 as an example, the amount of diodes necessary to achieve $1000 \mathrm{~lm}$ is calculated and shown in Table 2. Since the necessary input current for a $1000 \mathrm{~lm}$ system will be lower than the specified maximum, the diodes' lifetime will increase.

Table 2. Required amount of diodes and resulting input current for $1000 \mathrm{~lm}$

\begin{tabular}{|l|c|c|}
\hline \multicolumn{1}{|c|}{ Diode } & $\begin{array}{c}\text { Required Diodes for } \\
\mathbf{1 0 0 0} \mathbf{~} \mathbf{m} \text { at } \mathbf{T}_{\text {case }} \mathbf{= 2 5}{ }^{\circ} \mathbf{C}\end{array}$ & $\begin{array}{c}\text { Operating current per } \\
\text { Diode }\left(\mathbf{I}_{\mathbf{F}}\right)\end{array}$ \\
\hline OSLON Black Flat KW H2L531.TE & 2 & $720 \mathrm{~mA}$ \\
\hline OSRAM OSTAR LE UW U1A5 01 & 1 & $630 \mathrm{~mA}$ \\
\hline OSRAM PL TB450B (+ phosphor added) & 5 & $1200 \mathrm{~mA}$ \\
\hline
\end{tabular}

\section{SYSTEM DESIGN AND MODELING}

The system evaluated consists of a laser diode with $450 \mathrm{~nm}$ peak wavelength and an external power supply. The light output is focused on a remote phosphor layer which partially converts the blue light to light of higher wavelengths (480$650 \mathrm{~nm}$ ). This optical system can be regarded as a white light source. There is no thermal coupling between laser diodes and remote phosphor layer except for the optical beam. The case temperature of the laser diode is controlled to values between 25 and $50{ }^{\circ} \mathrm{C}$

\subsection{Thermal equivalent circuit diagram}

The thermal equivalent circuit diagram in Figure 3 is based on the assumption of a stationary system for the time period $d t$ without transient processes. The thermal eigentime constant $\lambda_{L D}$ of the laser diode has to be significantly smaller than $d t$. The electrical input power $\mathrm{P}_{-}$el is partly transferred as light energy $\mathrm{P}_{-}$opt_1 to the remote phosphor layer. The resulting optical power after passing the conversion layer is P_opt_2. The differences between P_el, P_opt_1 and P_opt_2 represent the power dissipation at each element. This power dissipation is lead away passing thermal resistances of the system's components. The thermal equivalent resistance between junction layer and case is Rjc, between case and environment Rcs and between conversion layer and environment R_con_a. 


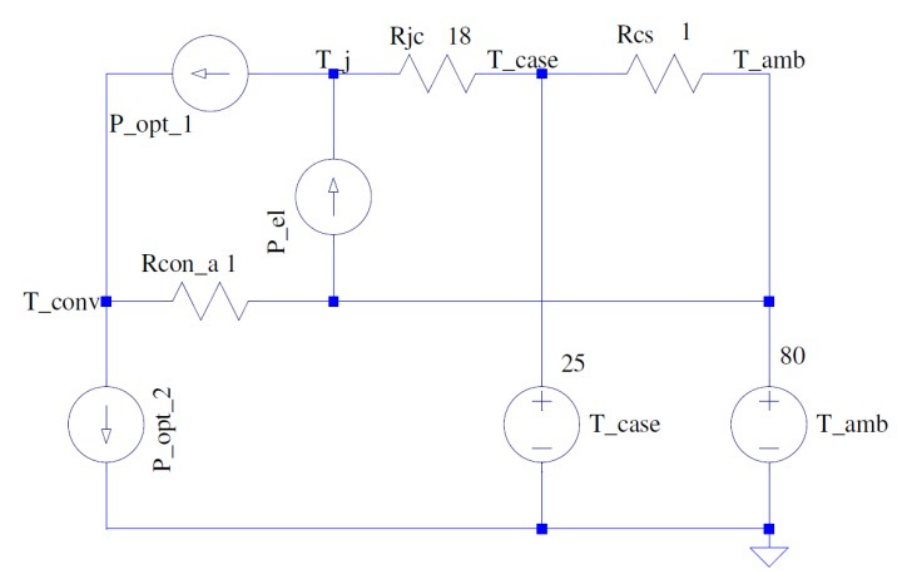

Figure 3. Thermal equivalent circuit diagram of the white light source consisting of several laser diodes and remote phosphor layer

\subsection{Modelling a laser based white light source with lifetime approximation}

The previously gathered system characteristics in combination with the thermal equivalent circuit diagram and Arrhenius' equation are combined to a Matlab Simulink model (Figure 4).

Input parameters are the diode's case temperature and the electrical current input. The measured characteristic maps are used as input data for the model blocks TIU and TIPopt1 to calculate $P_{-}$el, $P_{-}$opt_1 and the junction temperature $T_{-} j$. The junction temperature $T_{-} j$ is used according to equation (6) to estimate the diode's lifetime. The parameters case temperature and input current can be varied during the simulation to model different load cases.

Further elements are implemented to approximate the luminous flux and the wall plug efficiency of the whole system. In order to achieve a constant light output the input current of the system can be controlled. With these additions, the modeled system is very well approximated to the real situation in a vehicle.

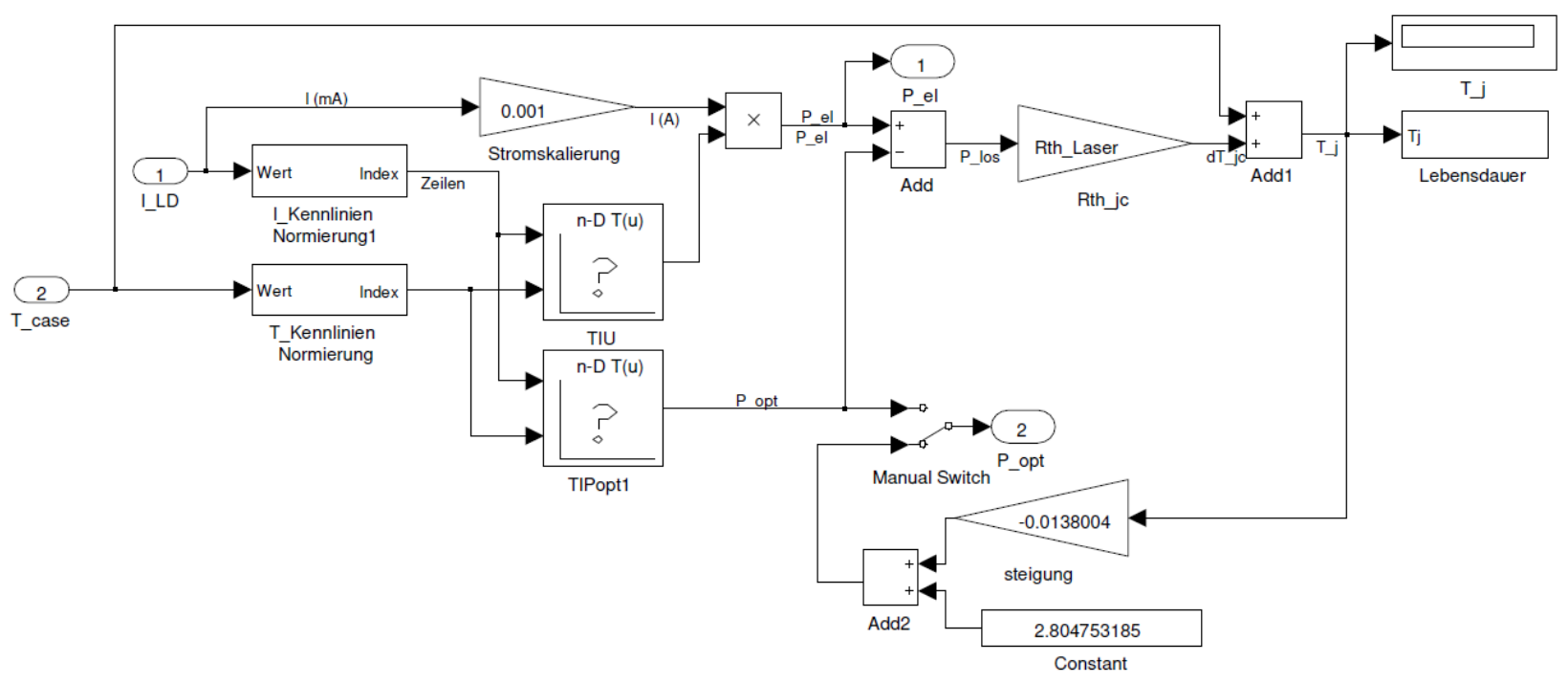

Figure 4. Characteristic curve based laser diode model with lifetime calculation for white light source 


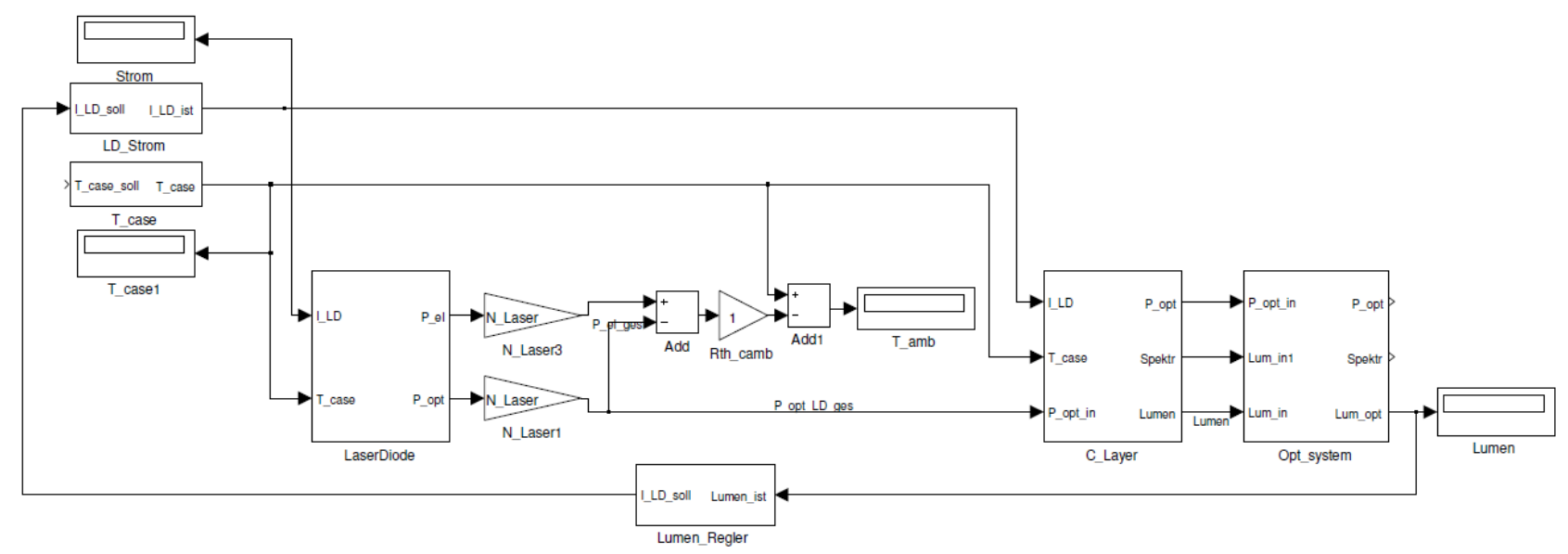

Figure 5. Light flux regulated model of laser based white light source

\subsection{Model parameters and setup}

In an experimental setup the system characteristics have been measured. A laser diode is placed opposed to a reflective phosphor layer inside an integrating sphere. The sphere is used to measure the optical power P_opt_2 for different case temperatures T_case and operating currents I_LD. To measure P_opt_1 a thermal power sensor head is used. A laser diode controller is used to generate and measure the electrical power $\mathrm{P}_{-}$el. The data is combined to a characteristic map of the diode's output power. Using a thermal imaging system the temperature of the phosphor layer is measured. Due to the phosphor layer being coated on a relatively large aluminum plate the temperature rise caused by the laser beam is quite low $(\leq 8 \mathrm{~K})$. Therefor its temperature as well as the conversion efficiency are considered to be constant for the thermal model and calculations.

The calculations of laser diode systems in the model are based on existing reliability assumptions of LEDs. Typically their lifetime is between 20000 and 50000 hours at a junction temperature of $\mathrm{Tj}=25^{\circ} \mathrm{C}$. The technical information provided by the LED's manufacturer indicate a value of 100 hours at $\mathrm{Tj}=175^{\circ} \mathrm{C}$. The activation energy can be calculated according to equation (1). Based on 50000 hours lifetime at $\mathrm{Tj}=25^{\circ} \mathrm{C}$, this leads to $\mathrm{E}_{\mathrm{A}}=0.477 \mathrm{eV}$. The activation energy of the laser diode modelled is considered to be of similar size.

\subsection{Simulation and analysis}

Using this approach the lifetime of the laser diodes can be calculated depending on the thermal resistance Rjc and the case temperature T_case (Figure 6). The required minimum lifetime of 1000 hours corresponds with a junction temperature of $100^{\circ} \mathrm{C}$.

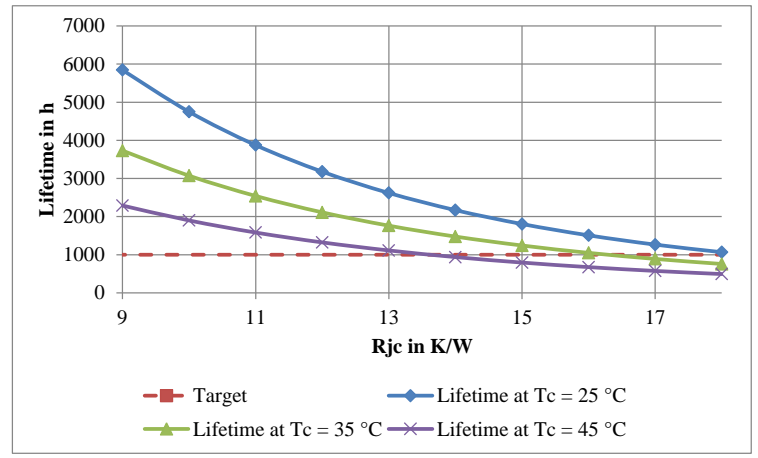

a

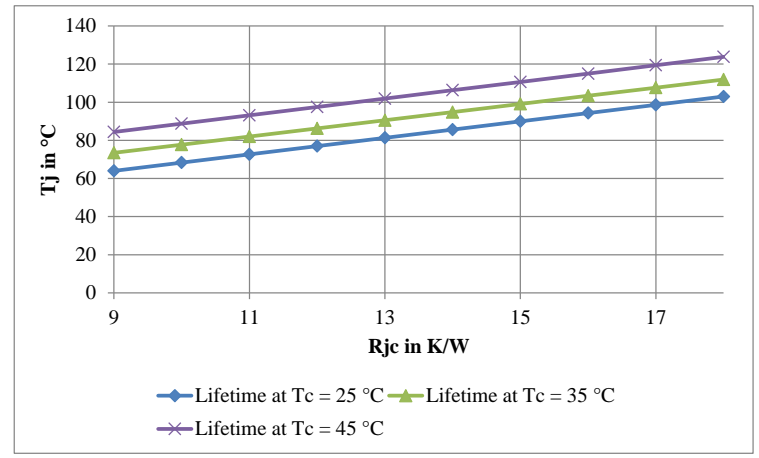

b

Figure 6. a) Simulation results for Lifetime during Rjc at 25.35 and $45^{\circ} \mathrm{C}$ and target lifetime. b) Calculated junction temperature during Rjc. 
As a result of higher temperatures the luminous flux is also reduced (Figure 2). At a case temperature of $50{ }^{\circ} \mathrm{C}$ for example, the luminous flux of the laser diode system is 146 lumens. To achieve higher luminous flux at this temperature, the input current of the diode has to be increased. As the diode is already driven at the maximum input current of $1200 \mathrm{~mA}$, this is not possible. For this reason the required amount of diodes in Table 2 has to be even higher. To be able to control the luminous flux of the diode system, the nominal laser current has to be lower than the maximum value. Considering this, 7 laser diodes each generating $146 \mathrm{~lm}$ are necessary to achieve the desired luminous flux of $1000 \mathrm{~lm}$.

Using the system model explained in section 4.2 the long term behavior of the system is analyzed for cyclic loads. This is done by activating the regulation of the output flux in the model and setting the target flux value to $146 \mathrm{~lm}$. The temperature of the laser diode is now varied between $25{ }^{\circ} \mathrm{C}$ and $50{ }^{\circ} \mathrm{C}$ using a sine function. This is done to simulate phases of warming up and cooling down during operation. As a result of this simulation, a lifetime of 949.5 hours can be estimated.

The diode system simulated in Figure 7 has a simulated lifetime close to the specified 1000 hours. To apply this approach to a real system e.g. a car's headlamp, additional influence factors have to be taken into consideration. Environment temperatures in this case are specified up to $80^{\circ} \mathrm{C}$. Without changes in the system's setup these temperatures lead to a lifetime reduction by factor 18 compared to an operation at $25^{\circ} \mathrm{C}$ (at Rjc $=18 \mathrm{~K} / \mathrm{W}$ ). So in this case an active cooling system is necessary to keep the system's lifetime. This leads to an increased power consumption and thus lowers the system's efficiency. Otherwise it would be necessary to reduce the thermal resistance between junction layer and case Rjc to a maximum of $4 \mathrm{~K} / \mathrm{W}$.
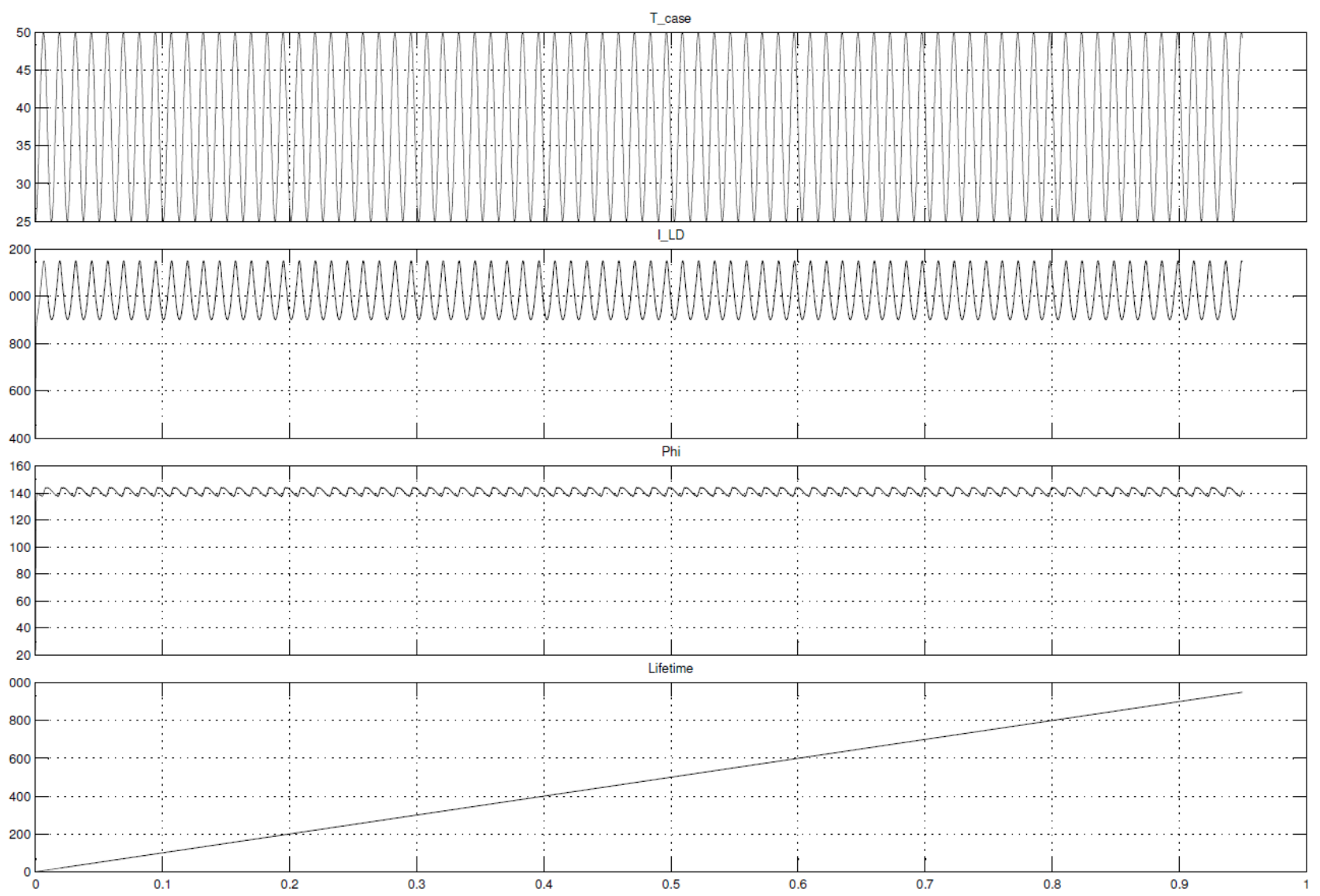

Figure 7. Simulation results for cycle T_case thermal Load and activated output flux Phi regulation to 146 lm. I_LD is the diode current during load cycles. Lifetime line shows the lifetime history/progress during the load. 


\section{CONCLUSIONS AND OUTLOOK}

In an experimental setup thermal effects on laser diode based white light sources have been evaluated. Based on these experimental data and information from LED data sheets, a lifetime simulation model was set up. This model is a first step to modelling and analyzing current state of the art of laser based white light sources. The simulation is able predict the diode's lifetime and the system's light output for specific applications and at any thermal load case.

In order to optimize the prediction of lifetime behavior, exact data for the reliability of laser diodes are necessary. Furthermore the reliability of the whole system has to be considered, i.e. power supply, cooling system and the diodes. The presented model proves that for automotive applications it is necessary to reduce the thermal resistance of laser diodes significantly in order to achieve long lifetimes. Resulting from lower thermal resistances inside laser diodes, the diode's efficiency and so the wall plug efficiency of the system can be significantly increased. Today's high power LEDs with thermal resistances of about $2 \mathrm{~K} / \mathrm{W}$ reach efficiencies of $90 \mathrm{~lm} / \mathrm{W}$. Apart from applications needing the high power density of a laser beam, the integration of diode based laser systems can only be useful if their efficiency reaches similar values.

\section{REFERENCES}

[1] Schubert, E. F., "Light-Emitting Diodes," second edition, Cambridge University Press, Cambridge (2010)

[2] Kloppenburg, G. and Lachmayer, R., "Development of a Laser Headlight," $13^{\text {th }}$ International Conference: Intelligent Automotive Lighting 2013, Wiesbaden, presentation (January 28 2013)

[3] Lachmayer, R. and Stephan, S., "Model-based validation and development of LED-systems: MValEnt", Proc. SPIE 8550, Optical Systems Design 2012, 855031 (December 18 2012)

[4] Glose, D., "Zuverlässigkeitsvorhersage für elektronische Komponenten unter mechanischer Belastung", Diplomica ${ }^{\circledR}$ Verlag GmbH, Hamburg (2009)

[5] OSRAM OS, "Data sheet PL-TB450B preliminary", June 12 2013, <http://www.osram-os.com/Graphics/XPic1/ 00088311_0.pdf/PL\%20TB450B.pdf> (December 12 2013)

[6] OSRAM OS, Data sheet "OSLON Black Flat KW H2L531.TE", May 14 2014, <http://www.osram-os.com/ Graphics/XPic7/00128042_0.pdf/KW\%20H2L531.TE\%20-\%20Oslon\%20Black\%20Flat.pdf> (July 14 2014)

[7] OSRAM OS, Data sheet "OSLON Black Flat LUW H9QP", February 18 2014, <http://www.osram-os.com/ Graphics/XPic8/00119025_0.pdf/LUW\%20H9QP\%20-\%20OSLON\%20Black\%20Flat.pdf> (July 14 2014)

[8] OSRAM OS, Data sheet "OSRAM OSTAR LE UW U1A3 01", June 17 2014, <http://www.osram-os.com/ Graphics/XPic7/00130643_0.pdf/LE\%20UW\%20U1A3\%2001\%20-\%20OSRAM\%20OSTAR\%20Headlamp \%20Pro.pdf> (July 292014 )

[9] OSRAM OS, Data sheet "OSRAM OSTAR LE UW U1A5 01", June 17 2014, <http://www.osram-os.com/ Graphics/XPic0/00130664_0.pdf/LE\%20UW\%20U1A5\%2001\%20-\%20OSRAM\%20OSTAR\%20Headlamp \%20Pro.pdf> (July 29 2014)

[10] PHILIPS, Data sheet "LUXEON Rebel", 2014, <www.philipslumileds.com/uploads/161/DS58-pdf> (July 30 2014) 